\title{
A mathematical determination of the pore size distribution and fractal dimension of a porous sample using spontaneous imbibition dynamics theory
}

\author{
Mumuni Amadu ${ }^{1} \cdot$ Michal J. Pegg ${ }^{1}$
}

Received: 14 February 2018 / Accepted: 10 May 2018 / Published online: 16 June 2018

(c) The Author(s) 2018

\begin{abstract}
Wallace sandstone has been extensively used by the construction industry for a long time in Nova Scotia. Apart from oxide analysis and a few strength-related parameter data found on some websites, petrophysical data regarding pore-sized distribution and fractal dimension are lacking. In the petroleum engineering literature, the spontaneous imbibition dynamics mechanism has been modeled where imbibition time has been linked to imbibition rise. One of the models links imbibition time to imbibition rise through a group of parameters that integrate the fractal dimension and sediment tortuosity. Based on the assumption of a bundle of parallel capillary tubes model found in the petrophysical literature, we have used the spontaneous imbibition model to derive an equation that links fractal dimension to porosity and permeability. Using literature source data on Wallace sandstone core samples, we have calculated the fractal dimension and pore size distribution using our equation. Results show that this sandstone has a significant level of heterogeneity. Calculation using another literature source data shows that our equation calculates fractal dimensions that are closer to those reported for the capillary pressure method. Although the assumption of bundle of parallel capillary tubes leads to deviations in calculated fractal dimensions using literature source data with experimentally determined porosity, permeability and fractal dimensions, our equation calculates meaningful values of fractal dimensions.
\end{abstract}

\section{Graphical abstract}

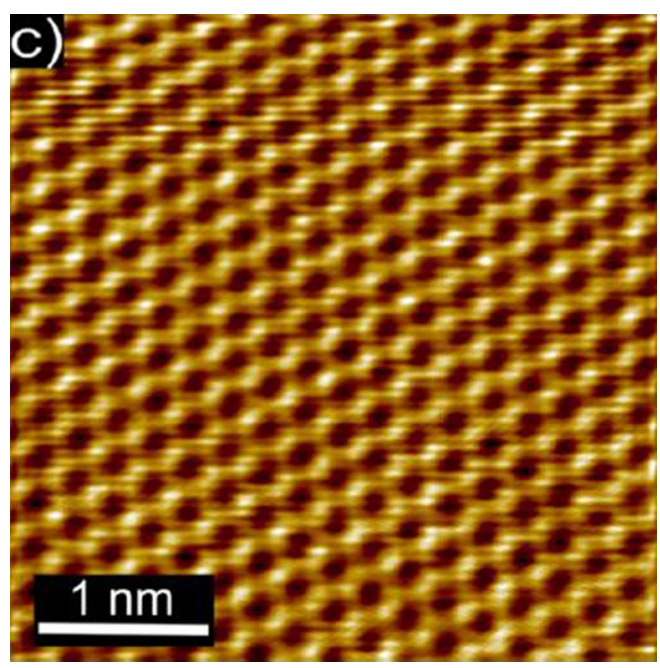

Keywords Pore size distribution $\cdot$ Fractal dimension $\cdot$ Spontaneous imbibition $\cdot$ Tortuosity $\cdot$ Heterogeneity $\cdot$ Homogeneity

Extended author information available on the last page of the article 


\section{List of symbols}

$A_{P} \quad$ Cross-sectional area of pore for spontaneous imbibition, $\mathrm{m}^{2}$

$D_{f} \quad$ Fractal dimension

$t \quad$ Time, s

$z \quad$ Imbibition distance, $m$

$\rho \quad$ Density of imbibing fluid, $\mathrm{kg} \mathrm{m}^{-3}$

$\mu \quad$ Dynamic viscosity of imbibing fluid, Pa.s

$\gamma_{L V} \quad$ Liquid-vapor interfacial tension, $\mathrm{Nm}^{-1}$

$\lambda_{\text {ind }} \quad$ Pore size distribution index

$\lambda_{\min }, \lambda_{\max }$ Parameters used in connection with fractal dimension

$\phi \quad$ Porosity-fraction

$\theta \quad$ Contact angle-degrees

$K \quad$ Absolute permeability, $\mathrm{m}^{2}$

\section{Introduction}

The pore size distribution of a porous material such as rock or a ceramic material is an important hydraulic parameter that has a bearing on several physical and physiochemical processes. For instance, the pore size distribution has a direct bearing on the strength of concrete (Kumar and Bhattacharjee 2003) and as such has a direct bearing on its ultimate strength such that the more the proportion of smaller pore sizes, the higher the strength. In the geotechnical literature, the microstructure of sedimentary rocks has been reported to affect its index properties (Sabatakakis et al. 2008). Zorlu et al. (2008) have predicted the uniaxial compressive strength of sandstones using petrographic-based models (Zorlu et al. 2008). Besides, the pore size distribution has an effect on drying shrinkage of activated concrete (Collins 2000).

Apart from the strength-related aspects of the pore size distribution of a porous material, hydraulic properties such as absolute permeability, relative permeability and capillary pressure characteristics are also dependent on pore size distribution (Jerauld and Salter 1990; Bennion 2006). Consequently, empirical relative permeability and capillary pressure functions presented by Brook and Corey (Brooks and Corey 1964) contain the pore size distribution index, which is a statistical measure characterizing the distribution of pore size in a given sample of a porous material.

The impact of pore size distribution on seismic velocity structure of the earth has been reported. In the geophysical literature, the fractal dimension which has a bearing on the pore size distribution index ( $\mathrm{Li} 2010$ ) has been reported to impart bimodal distributions of seismic velocities in rocks (Holliger and Lavender 1994).

Being a macroscopic pore structure parameter, pore size distribution will have a direct impact on the moisture absorption capacity of a facing porous rock such as sandstone slabs and, therefore, on its salt uptake and damage characteristics (Yu and Oguchi 2010). In Nova Scotia, Canada, Wallace sandstone has long been used as a building material, mostly as a facing stone. Consequently, knowledge of the pore size distribution characteristics of this sandstone is essential for the construction industry. What is more, with geological carbon storage being globally accepted as a technically and economically viable option for mitigating global warming (Wilson et al. 2008), knowledge of the effect of pore size distribution on the two-phase flow of anthropogenic carbon dioxide and formation brine is essential for future geological storage planning in Nova Scotia. Wallace sandstone is essentially glauconitic. However, while the glauconitic sandstones of Alberta have been studied (Bennion and Bachu 2008), Wallace sandstone has not yet received the desired attention from people in academia and the likes, apart from test data that was provided by the National Research Council of Nova Scotia (Council 1967). The mineral trapping potential of glauconitic sandstones in Alberta has been studied (Pruess et al. 2004). Therefore, the objective of the present study is to determine the pore size distribution index and the fractal dimension of this sandstone using spontaneous imbibition theory, as a first step towards getting an insight into the pore size distribution and fractal dimension of this geologic system. Different methods have been used for the determination of pore size distribution, ranging from nuclear magnetic resonance approach (Davies and Packer 1990) to capillary pressure methods (Pickell et al. 1996) (Pickell et al. 1996). In the present study, the theoretical basis of the spontaneous imbibition mechanism will be the preferred choice for the determination because of its simplicity.

\section{Theory}

Permeability, porosity and fractal dimension are among the macroscopic porous medium parameters (Dullien 2012). All these petrophysical parameters determine strength and hydraulic parameters of the porous medium and will, therefore, control spontaneous imbibition flow. In the literature, porosity has been linked to fractal dimension (Rieu and Sposito 1991). Absolute permeability has also been linked to fractal dimension (Yu and Cheng 2002). Thus, linking porosity and permeability to fractal dimension individually is an impetus for linking fractal dimension to porosity and permeability in a single equation. Therefore, the mathematical description of any hydraulic process of the porous medium that integrates porosity, permeability and fractal dimension in the desired combination can be exploited for developing such an equation.

Spontaneous imbibition is the spontaneous flow of a wetting fluid into a porous medium under capillary pressure 
gradient resulting from the combined effects of interfacial energies, pore structure and viscous forces.

In accordance with viscous non-compressible liquid flow along a cylindrical capillary tube, the application of Newton's dynamics gives (Zhmud et al. 2000):

$\rho\left[z z^{\prime \prime}+\left(z^{\prime}\right)^{2}\right]=\frac{2}{r} \gamma \cos \theta-\mu \frac{8}{r^{2}} \cos \theta * z z^{\prime}-\rho g z$

In this equation, $\rho$ is liquid density $\left[\mathrm{kg} \mathrm{m}^{-3}\right], z$ is the distance advanced by the spontaneous imbibition front $[\mathrm{m}]$, $z^{\prime \prime}$ is the acceleration $\left[\mathrm{ms}^{-2}\right], z^{\prime}=\mathrm{dz} / \mathrm{dt}$ is the velocity of the imbibition front $\left[\mathrm{ms}^{-1}\right], \theta$ is contact angle, $\gamma$ interfacial tension between imbibing and resident fluid, $r$ is radius of capillary tube $[\mathrm{m}], \mu$ dynamic viscosity of imbibing fluid $\left[\mathrm{N} \mathrm{sm}^{-2}\right]$ and $g$ is acceleration due to gravity $\left[\mathrm{ms}^{-2}\right]$.

The short-term asymptotic solution to this equation is given as:

$z(t)=\sqrt{\frac{r \gamma \cos \theta t}{2 \mu}}(t \rightarrow 0)$

The bundle of parallel tube model has proven to be useful in analysis of hydraulic problems associated with porous media (Dullien 2012; Kewen 2004) and can be applied to the process. This theory regards a porous sample to consist of a bundle of parallel capillary tubes of different sizes. Consequently, while the cross-sectional area opened to imbibition in a single capillary tube is simple and easy to quantify, the area opened to imbibition in a porous sample is given as (reference 40 of Cai et al. 2010):

$A_{\mathrm{P}}=\frac{\pi \lambda_{\max }^{2} D_{f}}{4\left(2-D_{f}\right)}(1-\phi)$

In this equation, $A_{\mathrm{P}}$ is area opened to imbibition flow, $\lambda_{\max }$ is maximum pore diameter, $D_{f}$ is fractal dimension of sediment and $\phi$ is porosity.

By considering the integral of imbibition volumes from minimum to maximum pore throat in addition to the tortuosity of pore throats and fractal dimension of the sediment, the distance imbibed versus time for early time periods is given as (Cai et al. 2010):

$$
\begin{aligned}
z^{2 D_{T}}= & \frac{\left(D_{T}+D_{f}-1\right)\left(2-D_{f}\right)}{D_{f}\left(2+D_{T}-D_{f}\right)}\left(\frac{\gamma \cos \theta}{4 \mu}\right) \\
& \left(\frac{\lambda_{\max } D_{T}}{\lambda_{\min }{ }^{1-D_{T}}}\right)\left(\frac{1-\beta^{2+D_{T}-D_{f}}}{1-\phi}\right) t
\end{aligned}
$$

where $D_{T}$ is tortuosity dimension of pore throat, and $D_{f}$ is the fractal dimension of sediment.

$$
\beta=\frac{\lambda_{\min }}{\lambda_{\max }}
$$

To seek the equivalent of Eq. (1) for imbibition flow into a porous medium requires invoking the bundle of parallel capillaries in the porous sample. Thus, for a straight capillary tube, the parameter $D_{T}$ is equal to 1 (Cai et al 2010). Thus, Eq. (9) becomes:

$$
\begin{gathered}
z^{2}=\frac{\left(D_{T}+D_{f}-1\right)\left(2-D_{f}\right)}{D_{f}\left(2+D_{T}-D_{f}\right)}\left(\frac{\gamma \cos \theta}{4 \mu}\right) \\
\left(\frac{\lambda_{\max } D_{T}}{\lambda_{\min }{ }^{1-D_{T}}}\right)\left(\frac{1-\beta^{2+D_{T}-D_{f}}}{1-\phi}\right) t
\end{gathered}
$$

Equation 4, for imbibition through a porous sample is the direct equivalent of Eq. (1) for imbibition through a capillary tube of known radius. It is interesting to see that in all equations, the driving force related to contact angle, surface tension and viscous shear force are present. Referring to Eq. (2), the following can be written in light of this observation:

$$
\begin{gathered}
\frac{\left(D_{T}+D_{f}-1\right)\left(2-D_{f}\right)}{D_{f}\left(2+D_{T}-D_{f}\right)}\left(\frac{\gamma \cos \theta}{4 \mu}\right)\left(\frac{\lambda_{\max }{ }^{D_{T}}}{\lambda_{\min }{ }^{1-D_{T}}}\right) \\
\left(\frac{1-\beta^{2+D_{T}-D_{f}}}{1-\phi}\right) \equiv\left(\frac{r \gamma \cos \theta}{2 \mu}\right)^{1 / 2}
\end{gathered}
$$

In this regard, all parameters on the left hand side except the contact angle, dynamic viscosity of imbibing fluid and interfacial tension account for an effective radius within the porous sample.

Equation 5 predicts a straight line between the square of imbibition rise and imbibition time. The gradient of the plot will be given as:

$$
\begin{aligned}
\frac{\mathrm{d} z^{2}}{\mathrm{~d} t}= & \frac{\left(D_{T}+D_{f}-1\right)\left(2-D_{f}\right)}{D_{f}\left(2+D_{T}-D_{f}\right)}\left(\frac{\gamma \cos \theta}{4 \mu}\right) \\
& \left(\frac{\lambda_{\max } D_{T}}{\lambda_{\min } 1-D_{T}}\right)\left(\frac{1-\beta^{2+D_{T}-D_{f}}}{1-\phi}\right)
\end{aligned}
$$

To exploit this for achieving the principal objective of this paper, an equivalent radius of the porous system used in Leveret $\mathrm{J}$ function will be defined as (Gostick et al. 2006):

$r_{e}=\sqrt{\frac{K}{\phi}}$

where $r_{e}$ is equivalent radius, $K$ is absolute permeability and $\phi$ is porosity-fraction.

Setting

$$
\eta=\frac{\left(D_{T}+D_{f}-1\right)\left(2-D_{f}\right)}{D_{f}\left(2+D_{T}-D_{f}\right)}\left(\frac{1}{4 \mu}\right)\left(\frac{\lambda_{\max }{ }^{D_{T}}}{\lambda_{\min }{ }^{1-D_{T}}}\right)\left(\frac{1-\beta^{2+D_{T}-D_{f}}}{1-\phi}\right)
$$


$\frac{\mathrm{d} z^{2}}{\mathrm{~d} t}=\eta \gamma \cos \theta$

Equation (2) can be written in terms of the equivalent radius as:

$z(t)=\sqrt{\frac{K}{\phi}} \sqrt{\frac{\gamma \cos \theta * t}{2 \mu}}(t \rightarrow 0)$

This gives the gradient of the plot as:

$\frac{\mathrm{d} z^{2}}{\mathrm{~d} t}=\sqrt{\frac{K}{\phi}} \frac{\gamma \cos \theta}{2 \mu}$

From this, the contact angle for the porous medium is calculated as:

$\cos \theta=\left(\frac{K}{\phi}\right)^{-0.5} \frac{2 \mu}{\gamma} \frac{\mathrm{d}\left(z^{2}\right)}{\mathrm{d} t}$

Substitution into Eq. (10) gives:

$\frac{d z^{2}}{d t}=\eta \gamma\left(\frac{K}{\phi}\right)^{-0.5} \frac{2 \mu}{\gamma} \frac{d z^{2}}{d t}$

$\Leftrightarrow 2 \eta \mu\left(\frac{K}{\phi}\right)^{-0.5}=1$

A simplified form of this equation can be sought using the bundle of parallel capillary tube model where tortuosity $\left(D_{T}\right)$ is unity. Thus for $D_{T}=1$ :

$\eta=\frac{\left(D_{T}+D_{f}-1\right)\left(2-D_{f}\right)}{D_{f}\left(3-D_{f}\right)}\left(\frac{1}{4 \mu}\right)\left(\frac{\lambda_{\max }^{1}}{\lambda_{\min }^{1-1}}\right)\left(\frac{1-\beta^{3-D_{f}}}{1-\phi}\right)$

Hence:

$\frac{\left(D_{f}\right)\left(2-D_{f}\right)}{D_{f}\left(3-D_{f}\right)}\left(\frac{1}{2}\right)\left(\lambda_{\max }\right)\left(\frac{1-\beta^{3-D_{f}}}{1-\phi}\right)\left(\frac{K}{\phi}\right)^{-0.5}=1$

Hence:

$\frac{\left(2-D_{f}\right)}{\left(3-D_{f}\right)}\left(\frac{1-\beta^{3-D_{f}}}{1-\phi}\right)=2 \lambda_{\max }^{-1}\left(\frac{K}{\phi}\right)^{0.5}$

The right-hand side of this equation has no unit because the reciprocal of length multiplied by length cancels. This agrees with the left-hand side, which is fractal dimension. Porosity is in fraction and the unit of $\beta$ is dimensionless.

$\beta=0.01$, (Cai et al. 2010).

Hence:
$\frac{\left(2-D_{f}\right)}{\left(3-D_{f}\right)}\left(\frac{1-0.01^{3-D_{f}}}{1-\phi}\right)=2 \lambda_{\max }^{-1}\left(\frac{K}{\phi}\right)^{0.5}$

Characterization of the porous sample enables determination of absolute permeability and porosity. The maximum pore diameter $\lambda_{\max 1}$ is calculated based on a model of an equilateral triangle arrangement (reference $38 \mathrm{Cai}$ et al. 2010), while $\lambda_{\max 2}$, is calculated based on a model of a square arrangement of circular particles (reference 44 of Cai et al. 2010).

The parameter, $\lambda_{\max }$, is calculated as (Cai et al. 2010):

$\lambda_{\max }=\frac{\lambda_{\max 1}+\lambda_{\max 2}}{2}=\frac{\frac{D_{s}}{2} \sqrt{\frac{2 \phi}{1-\phi}}+\frac{D_{s}}{2}\left[\sqrt{\frac{\phi}{1-\phi}}+\sqrt{\frac{\pi}{4(1-\phi)}}\right]}{2}$

where $D_{s}$ is grain diameter [cm].

This parameter can be substituted into Eq. (18). Using the definition of mean pore radius (Eq. 8), the resulting equation is such that only the fractal dimension is unknown where porosity is given. This is an algebraic equation that can be solved for the fractal dimension of the sediment. The equation will be written as:

$$
\begin{gathered}
\frac{\left(2-D_{f}\right)}{\left(3-D_{f}\right)}\left(\frac{1-0.01^{3-D_{f}}}{(1-\theta)}\right)=2\left[\left(\frac{2 \phi}{1-\phi}\right)^{0.5}\right. \\
\left.+\left\{\left(\frac{\phi}{1-\phi}\right)^{0.5}+\left(\frac{\pi}{4(1-\phi)}\right)^{0.5}\right\}\right]
\end{gathered}
$$

The relationship between the fractal dimension and the pore size distribution index for a three-dimensional system is given as (Kewen 2004):

$\lambda=3-D_{f}$

where $\lambda$ is the pore size distribution index.

Solving for pore size distribution and substituting into Eq. (22) gives:

$$
\begin{aligned}
& \frac{(\lambda-1)}{(\lambda)}\left(\frac{1-0.01^{\lambda}}{(1-\theta)}\right)=2\left[\left(\frac{2 \phi}{1-\phi}\right)^{0.5}\right. \\
& \left.+\left\{\left(\frac{\phi}{1-\phi}\right)^{0.5}+\left(\frac{\pi}{4(1-\phi)}\right)^{0.5}\right\}\right]
\end{aligned}
$$

Equation (22) links fractal dimension to porosity as found in another published research work, where fractal dimension has been shown to be a function of porosity and connectivity (Pothuaud et al. 2000).

Thus, the petrophysical characterization of samples of Wallace sandstone enables the determinations of porosity and absolute permeability and the substitution of these 
together with $\lambda_{\max }$ enables solution for the fractal dimension of this sandstone. Also, porosity data of this sandstone can be substituted into Eq. (24) to solve for the pore size distribution index.

\section{Derivations of fractal dimension}

To solve Eq. (18) for the fractal dimension of Wallace sandstone requires testing its suitability. This requires using literature data containing porosity and permeability with corresponding fractal dimensions. In solving this equation, we wrote an objective function as:

$f\left(D_{f}\right)=\frac{\left(2-D_{f}\right)}{\left(3-D_{f}\right)}\left(\frac{1-0.01^{3-D_{f}}}{1-\phi}\right)-2 \lambda_{\max }^{-1}\left(\frac{K}{\phi}\right)^{0.5}$

This was written as a formula in excel. The value of this objective function was set to 0 and the fractal dimension was varied by the solver program until the value was close to zero as much as possible. The value of the fractal dimension was then obtained.

Zhang and Weller (2014) studied the fractal dimension of sandstone samples from the Eocene sandstone formation in China. Table 6 of Appendix 1 at the end of this paper shows data on porosities, permeabilities and fractal dimensions of corresponding samples using capillary pressure, nuclear magnetic resonance and specific surface area methods. Using Eq. (18), the fractal dimensions for different samples in the appendix have been calculated and compared to those reported in the appendix. Following this, porosity and permeability data for Wallace sandstone have been extracted from literature source (Tables 7 and 8 of Appendix 2) for the calculation of the fractal dimensions. Accordingly, mean pore radii were calculated using porosity and permeability data in accordance with Eq. (8).

\section{Results and discussion}

Table 1 shows results of calculated fractal dimensions of samples with petrophysical data from Table 6 of Appendix 1. Column 7 of the table shows actual fractal dimensions calculated using Eq. (18) of the present work. Generally, Eq. (18) calculates fractal dimension below values indicated by the maximum values of nuclear magnetic resonance (NMR), and above values indicated by other methods. A better idea about the accuracy of the spontaneous imbibition

Table 2 Deviations of fractal dimensions from calculated fractal in the present study

\begin{tabular}{llll}
\hline CP & NMR max & NMR min & SS \\
\hline 0.42 & 0.35 & -0.34 & -0.28 \\
-0.20 & 0.28 & -0.24 & -0.20 \\
-0.23 & 0.27 & -0.09 & -0.23 \\
-0.34 & 0.23 & -0.29 & -0.34 \\
-0.26 & 0.22 & -0.02 & -0.26 \\
-0.22 & 0.36 & -0.36 & -0.22 \\
-0.17 & 0.32 & -0.33 & -0.17 \\
-0.14 & 0.42 & -0.32 & -0.14 \\
-0.09 & 0.35 & -0.37 & -0.09 \\
-0.11 & 0.43 & -0.39 & -0.11 \\
-0.18 & 0.39 & -0.31 & -0.18 \\
-0.14 & 0.39 & -0.29 & -0.14 \\
\hline
\end{tabular}

Table 1 Comparison of calculated fractal dimensions with literature values from Table 6 in Appendix 1 (converted to 3 decimal places)

\begin{tabular}{lllllll}
\hline Permeability & Porosity & $\begin{array}{l}\text { Fractal dimension } \\
\text { from capillary pres- } \\
\text { sure } \\
\text { (CP) }\end{array}$ & $\begin{array}{l}\text { Fractal dimension } \\
\text { from nuclear magnetic } \\
\text { resonance } \\
\text { (NMR) max }\end{array}$ & $\begin{array}{l}\text { Fractal dimension } \\
\text { from nuclear magnetic } \\
\text { resonance } \\
\text { (NMR) min }\end{array}$ & $\begin{array}{l}\text { Fractal dimension } \\
\text { from specific internal } \\
\text { surface }\end{array}$ & $\begin{array}{l}\text { Calculated fractal } \\
\text { dimension in the } \\
\text { present work }\end{array}$ \\
\hline 0.129 & 0.125 & 2.984 & 2.908 & 2.224 & 2.277 & 2.560 \\
18.279 & 0.166 & 2.337 & 2.817 & 2.296 & 2.337 & 2.535 \\
25.056 & 0.17 & 2.305 & 2.803 & 2.438 & 2.305 & 2.533 \\
60.502 & 0.162 & 2.196 & 2.77 & 2.248 & 2.196 & 2.536 \\
45.68 & 0.163 & 2.274 & 2.753 & 2.515 & 2.274 & 2.537 \\
0.081 & 0.137 & 2.337 & 2.914 & 2.190 & 2.337 & 2.554 \\
0.13 & 0.139 & 2.384 & 2.875 & 2.226 & 2.413 & 2.553 \\
0.227 & 0.147 & 2.413 & 2.968 & 2.231 & 2.468 & 2.550 \\
0.364 & 0.132 & 2.465 & 2.910 & 2.188 & 2.451 & 2.558 \\
0.432 & 0.143 & 2.451 & 2.991 & 2.169 & 2.364 & 2.550 \\
0.624 & 0.156 & 2.364 & 2.934 & 2.934 & 2.256 & 2.405 \\
1.297 & 0.15 & 2.405 & & &
\end{tabular}

max maximum value, min minimum value 
approach in the present study can be obtained by calculating the deviations from reported fractal dimensions shown in Table 6 of Appendix 1.

Table 2 shows calculated deviations, where deviation is measured as the difference between reported fractal dimension and calculated fractal dimension in this work. Overall, fractal dimensions calculated using Eq. (18) show less deviations compared to the capillary pressure method. This means predictions of fractal dimension using Eq. (18) are closer to values of the capillary pressure method.

Table 3 shows the calculated fractional deviations using Eq. (18). This table again shows that Eq. (18) calculates fractal dimensions closer to those reported for the capillary pressure method and this is clearly seen in Table 1.

In the derivation of Eq. (18), the assumption of bundle of parallel capillary tubes with varying radii was the basis. However, in reality, the effect of overburden compaction in the sedimentary column and the attendant pressure solution effect (Yang 2000) suggest real sediments will have significant levels of tortuosity. This means the parameter, $D_{T}$, corresponding to the tortuosity fractal dimension, which measures the convolutedness of the capillary path ways in Eq. (4) is an essential parameter. What is more, the effect of sediment deformation under stress heterogeneity and grain size evolution (Iverson et al. 1996) is to cause collapse of original large pore spaces leading to varied pore size distribution, which causes higher fractal dimensions or heterogeneities. In the published work of Zhang and Weller (2014), (Table 6 in Appendix 1), the age of the formation is Eocene and the original depths of the samples are between 3332 and 3738 meters. Under such overburden conditions, the tortuosity factor of the sediment cannot be neglected. Therefore, any model that neglects tortuosity will obviously calculate fractal dimensions different from actual or expected values. That is why our assumption of bundle of parallel capillary tubes without tortuosity has led to calculated fractal dimensions

Table 3 Fractional deviations from calculated values

\begin{tabular}{llll}
\hline CP & NMR max & NMR min & SSA \\
\hline 0.14 & 0.12 & -0.15 & -0.12 \\
-0.09 & 0.10 & -0.10 & -0.09 \\
-0.10 & 0.10 & -0.04 & -0.10 \\
-0.15 & 0.08 & -0.13 & -0.15 \\
-0.12 & 0.08 & -0.01 & -0.12 \\
-0.09 & 0.12 & -0.16 & -0.09 \\
-0.07 & 0.11 & -0.14 & -0.07 \\
-0.05 & 0.14 & -0.14 & -0.05 \\
-0.04 & 0.12 & -0.17 & -0.04 \\
-0.04 & 0.15 & -0.18 & -0.04 \\
-0.05 & 0.16 & -0.10 & -0.05 \\
-0.06 & 0.13 & -0.13 & -0.06 \\
\hline
\end{tabular}

with deviation, though values of calculated fractal dimensions fall within those expected for sandstones.

Comparing calculated values of fractal dimensions using Eq. (18) to those reported for the capillary pressure method in Table 1 gave impetus for calculation of the fractal dimension of Wallace sandstone samples using this equation. This is because we have data on absolute permeability, which is correlated with pore size distribution generated from capillary pressure data (Marshall 1958). Zakir Ullah (2013) studied the effect of waste cooking oil on rock wettability in his graduation project work at Dalhousie University (Ullah 2013). He used four core samples of Wallace sandstone. Tables 7 and 8 in Appendix 2give the details of characterization results of this sandstone samples under ambient conditions.

Accordingly, Table 4 shows fractal dimensions of Wallace sandstone calculated using Eq. (18). The mean value is 2.550. Table 5 shows results of pore size distribution index calculation using Eq. (21) and values of fractal dimensions from Table 4. Accordingly, values of pore size distribution index with a mean of 0.446 fall within those reported elsewhere for sandstones (Bloomfield et al. 2001).

All values of fractal dimensions calculated using Eq. (18) found in Table 1 and Table 3 fall within those for sandstones that have been reported by other workers (Zhang and Weller 2014; Radlinski et al. 2004; Dathe et al. 2001). This testifies to the suitability of this equation.

Apart from its relationship to varying physiochemical processes reviewed in Sect. 1, the fractal dimension and pore size distribution give indication about the heterogeneities or homogeneities of sediments (Pfeifer and Avnir 1983; Wheatcraft and Tyler 1988). Accordingly, the bigger the fractal dimension of a sediment, the more heterogeneous it is and vice versa. Bigger values of fractal dimensions will have wider values of wetting phase saturation and a

Table 4 Calculated fractal dimensions for Wallace sandstone core samples using Eq. (18)

\begin{tabular}{lllll}
\hline Sample 1 & Sample 2 & Sample 3 & Sample 4 & Mean value \\
\hline 2.564 & 2.553 & 2.549 & 2.550 & 2.550 \\
\hline
\end{tabular}

Table 5 Fractal dimension and pore size distribution index

\begin{tabular}{lll}
\hline Sample no. & Fractal dimension & $\begin{array}{l}\text { Pore size } \\
\text { distribution } \\
\text { index }\end{array}$ \\
\hline Sample 1 & 2.564 & 0.436 \\
Sample 2 & 2.553 & 0.447 \\
Sample 3 & 2.549 & 0.451 \\
Sample 4 & 2.550 & 0.450 \\
\hline
\end{tabular}


broader range of capillary pressure data, whereas smaller values will be characterized by bigger values of pore size distribution as given by Eq. (21). Such rocks are characterized by narrow ranges of capillary pressure and wetting phase saturation. From Tables 4 and 5, the mean values of fractal dimension and pore size distribution index are 2.554 and 0.446 , respectively. In the literature, typical values of pore size distribution vary from 0.5 for materials with larger range of pore sizes with a value of 0.45 being characteristic of consolidated formations (Bloomfield et al. 2001). Therefore, values calculated for Wallace sandstone in this paper are characteristic of eminent heterogeneity which is further supported by oxide analysis. The oxide analysis shows $82 \%$ (Quary 2001) of silica and varying compositions of other minerals and these point to heterogeneity in mineralogy, which reflects the pore size distribution index calculated in our work. Based on evidence of deviations of fractal dimension reported in Table 1, we admit that calculation of the fractal dimension of Wallace sandstone reported in Table 4 also has this deviation, but calculated fractal dimensions are appreciable because they reflect those of sandstones.

\section{Conclusion}

The fractal dimension and pore size distribution of a sediment is an important petrophysical parameter that has a strong and direct bearing on its hydraulic and physiochemical properties. In Canada, the glauconitic sandstones of Alberta have been studied and data for petrophysical parameters exist. In Nova Scotia, Canada, Wallace sandstones are glauconitic sandstones that have been used by the construction industries for long. Apart from test data on mechanical strength and reports of oxide compositions of this sandstone, no data on pore size distribution and fractal dimension exist to the best of our knowledge. We have used analytical approach based on spontaneous imbibition dynamics to derive an equation that integrates pore size distribution, porosity and permeability of sediment. In the application of this equation to Wallace sandstone, our principal assumption was that a cylindrical sample of Wallace sandstones core sample can be regarded as a bundle of parallel capillary tubes without tortuosity. Based on this equation, we have calculated the fractal dimension and pore size distribution index for this sandstone, hoping that it will provide an insight into its hydraulic and petrophysical characteristics. The following sums up the conclusion of this study:

1. Wallace sandstone has a fractal dimension with a mean value of 2.554 and a pore size distribution index with a mean value of 0.446 ;

2. Values of pore size distribution and fractal dimension deduced in this study show that this sandstone is characterized by a significant level of heterogeneity and it will, therefore, exhibit a wider range of capillary pressure versus wetting phase saturation;

3. Mean values of pore size distribution index and fractal dimension calculated in this study fall within those reported in the literature for sandstones;

4. Our assumption of bundle of parallel capillary tubes for sediment samples has led to deviations of fractal dimensions from a literature source data but deviations are not alarming.

\section{Future work}

Our observation that the bundle of parallel capillary tubes of varying radius assumption leads to calculation of fractal dimension with deviations has motivated future work. In this regard, our objective is to use a more rigorous and robust mathematical model that will integrate the pore tortuosity dimension into the spontaneous imbibition model. This will enable us to deduce more representative values of fractal dimension and pore size distribution index of Wallace sandstone.

Acknowledgements We wish to acknowledge the immense support of Dalhousie University Document Delivery section for their timely supply of requested literature materials. The timely completion of our manuscript would not have been possible without this support.

Open Access This article is distributed under the terms of the Creative Commons Attribution 4.0 International License (http://creativeco mmons.org/licenses/by/4.0/), which permits unrestricted use, distribution, and reproduction in any medium, provided you give appropriate credit to the original author(s) and the source, provide a link to the Creative Commons license, and indicate if changes were made.

\section{Appendix 1}

See Table 6. 
Table 6 Data from literature source (Zhang and Weller 2014)

\begin{tabular}{llllll}
\hline Permeability & Porosity & $\begin{array}{l}\text { Fractal dimension from } \\
\text { capillary pressure } \\
\text { CP }\end{array}$ & $\begin{array}{l}\text { Fractal dimension from } \\
\text { nuclear magnetic resonance } \\
\text { (NMR) max }\end{array}$ & $\begin{array}{l}\text { Fractal dimension from } \\
\text { nuclear magnetic resonance } \\
\text { (NMR) min }\end{array}$ & $\begin{array}{l}\text { Fractal dimension from } \\
\text { specific internal surface }\end{array}$ \\
\hline 0.129 & 0.125 & 2.984 & 2.908 & 2.224 & 2.277 \\
18.279 & 0.166 & 2.337 & 2.817 & 2.296 & 2.337 \\
25.056 & 0.17 & 2.305 & 2.803 & 2.438 & 2.305 \\
60.502 & 0.162 & 2.196 & 2.77 & 2.248 & 2.196 \\
45.68 & 0.163 & 2.274 & 2.753 & 2.515 & 2.274 \\
0.081 & 0.137 & 2.337 & 2.914 & 2.190 & 2.337 \\
0.13 & 0.139 & 2.384 & 2.875 & 2.226 & 2.384 \\
0.227 & 0.147 & 2.413 & 2.968 & 2.231 & 2.413 \\
0.364 & 0.132 & 2.465 & 2.910 & 2.188 & 2.468 \\
0.432 & 0.143 & 2.451 & 2.991 & 2.169 & 2.451 \\
0.624 & 0.156 & 2.364 & 2.934 & 2.237 & 2.364 \\
1.297 & 0.15 & 2.405 & 2.934 & 2.256 & 2.405 \\
\hline
\end{tabular}

\section{Appendix 2}

See Tables 7 and 8 .

Table 7 Petrophysical characteristics of core samples (Ullah 2013): Core dimensions

\begin{tabular}{llll}
\hline Sample no. & Length, cm & Diameter, cm & $\begin{array}{l}\text { Bulk } \\
\text { volume, } \\
\mathrm{cm}^{3}\end{array}$ \\
\hline Sample 1 & 7.16 & 3.78 & 80.30 \\
Sample 2 & 7.20 & 3.78 & 80.75 \\
Sample 3 & 7.14 & 3.78 & 80.08 \\
Sample 4 & 7.14 & 3.78 & 80.28 \\
\hline
\end{tabular}

Table 8 Test results (Ullah 2013)

\begin{tabular}{lllllll}
\hline Sample & Dry weight gm & Wet weight gm & Porosity $\%$ & $\begin{array}{l}\text { Permeabil- } \\
\text { ity } \mathrm{mD}\end{array}$ & $\begin{array}{l}\text { Permeability, } \\
10^{-12} \mathrm{~m}^{2}\end{array}$ & $\begin{array}{l}\text { Mean } \\
\text { radius, } \\
10^{-6} \mathrm{~m}\end{array}$ \\
\hline 1 & 187 & 199 & 15.55 & 1.88 & 1.86 & 3.49 \\
2 & 188 & 199 & 13.85 & 1.89 & 1.87 & 3.67 \\
3 & 185 & 197 & 14.55 & 1.85 & 1.83 & 3.54 \\
4 & 187 & 199 & 14.40 & 1.84 & 1.82 & 3.55 \\
\hline
\end{tabular}

\section{References}

Bennion DB (2006) The impact of interfacial tension and pore size distribution/capillary pressure character on $\mathrm{CO} 2$ relative permeability at reservoir conditions in CO2-brine systems. In: SPE/ DOE Symposium on Improved Oil Recovery. Society of Petroleum Engineers

Bennion B, Bachu S (2008) Drainage and imbibition relative permeability relationships for supercritical $\mathrm{CO} 2 /$ brine and $\mathrm{H} 2 \mathrm{~S} / \mathrm{brine}$ systems in intergranular sandstone, carbonate, shale, and anhydrite rocks. SPE Reservoir Eval Eng 11(3):487-496

Bloomfield J, Gooddy D, Bright M, Williams P (2001) Pore-throat size distributions in Permo-Triassic sandstones from the United Kingdom and some implications for contaminant hydrogeology. Hydrogeol J 9(3):219-230

Brooks R, Corey T (1964) Hydraulic Properties Of Porous Media. Hydrology Papers

Cai J, Yu B, Zou M, Luo L (2010) Fractal characterization of spontaneous co-current imbibition in porous media. Energy Fuels $1860-1867$ 
Collins FJ (2000) Effect of pore size distribution on drying shrinking of alkali-activated slag concrete. Cem Concr Res 30(9):1401-1406

Council NR (1967) Results ASTM testing. http://www.wallacequa rries.com/test-data.html. http://www.wallacequarries.com/testdata.html

Dathe A, Eins S, Niemeyer J, Gerold G (2001) The surface fractal dimension of the soil-pore interface as measured by image analysis. Geoderma 103(1):203-229

Davies S, Packer KJ (1990) Pore-size distributions from nuclear magnetic resonance spin-lattice relaxation measurements of fluidsaturated porous solids. I. Theory and simulation. J Appl Phys 67(6):3163-3170

Dullien FA (2012) Porous media: fluid transport and pore structure. Academic press

Gostick JT, Pritzker MD, Volfkovich YM, Sakars A (2006) Capillary pressure and hydrophilic porosity in gas diffusion layers for polymer electrolyte fuel cells. J Power Sources 156(2):375-387

Holliger K, Levander A (1994) Structure and seismic response of extended continental crust: stochastic analysis of the Strona-Ceneri and Ivrea zones, Italy. Geology 22(1):79-82

Iverson NR, Hooyer TS, Hooke RL (1996) A laboratory study of sediment deformation: stress heterogeneity and grain-size evolution. Ann Glaciol, 167-175

Jerauld GR, Salter SJ (1990) The effect of pore-structure on hysteresis in relative permeability and capillary pressure: pore-level modeling. Transport in porous media. Transport in porous media 5(2):103-151

Kewen L (2004) Theoretical development of the Brooks-Corey capillary pressure model from fractal modeling of porous media. In: SPE/DOE Symposium on Improved Oil Recovery. Society of Petroleum Engineers

Kumar R, Bhattacharjee B (2003) Porosity, pore size distribution and in situ strength of concrete. Cement Conc Res 33(1):155-164

Li K (2010) Analytical derivation of Brooks-Corey type capillary pressure models using fractal geometry and evaluation of rock heterogeneity. J Petrol Sci Eng 73(1):20-26

Marshall TJ (1958) A relation between permeability and size distribution of pores. Eur J Soil Sci 9(1):1-8

Pfeifer P, Avnir D (1983) Chemistry in noninteger dimensions between two and three. I. Fractal theory of heterogeneous surfaces. J Chem Phys 79(7):3558-3565

Pickell JJ, Swanson BF, Hickman WB (1996) Application of air-mercury and oil-air capillary pressure data in the study of pore structure and fluid distribution. Soc Petrol Eng J 60(1):55-61
Pothuaud L, Benhamou CL, Porion P, Lespessailles E, Harba R, Levitz P (2000) Fractal dimension of trabecular bone projection texture is related to three-dimensional microarchitecture. J Bone Miner Res 15(4):691-699

Pruess K, García J, Kovscek T, Oldenburg C, Rutqvist J, Steefel C, $\mathrm{Xu} \mathrm{T}$ (2004) Code intercomparison builds confidence in numerical simulation models for geologic disposal of $\mathrm{CO}_{2}$. Energy 29(9):1431-1444

Quary WS (2001) http://www.wallacequarries.com. Retrieved from Wallacde Quarry

Radlinski AP, Ioannidis MA, Hinde AL, Hainbuchner M, Baron M, Rauch H, Kline SR (2004) Angstrom-to-millimeter characterization of sedimentary rock microstructure. J Colloid Interface Sci 274(2):607-612

Rieu M, Sposito G (1991) Fractal fragmentation, soil porosity, and soil water properties I. Theory. Soil Sci Soc Am J 55(5):1231-1238

Sabatakakis N, Koukis G, Tsiambaos G, Papanakli S (2008) Index properties and strength variation controlled by microstructure for sedimentary rocks. Eng Geol 97(1):80-90

Ullah Z (2013) The effect of waste cooking oil on wettability

Wheatcraft SW, Tyler SW (1988) An explanation of scale-dependent dispersivity in heterogeneous aquifers using concepts of fractal geometry. Water Resour Res 24(4):566-578

Wilson EJ, Morgan MG, Apt JB, Bunting C, Gode J, Pollak MF (2008) Regulating the geological sequestration of $\mathrm{CO}_{2}$. ACS Publications

Yang XS (2000) Pressure solution in sedimentary basins: effect of temperature gradient. Earth Planet Sci Lett 176(2):233-243

Yu B, Cheng P (2002) A fractal permeability model for bi-dispersed porous media. Int J Heat Mass Transf 45(14):2983-2993

Yu S, Oguchi CT (2010) Role of pore size distribution in salt uptake, damage, and predicting salt susceptibility of eight types of Japanese building stones. Eng Geol 115(3):226-236

Zhang Z, Weller A (2014) Fractal dimension of pore-space geometry of an Eocene sandstone formation. Geophysics 79(6):D377-D387

Zhmud BV, Tiberg F, Hallstensson K (2000) Dynamics of capillary rise. J Colloid Interface Sci 228(2):263-269

Zorlu K, Gokceoglu C, Ocakoglu F, Nefeslioglu HA, Acikalin S (2008) Prediction of uniaxial compressive strength of sandstones using petrography-based models. Eng Geol 141-158

Publisher's Note Springer Nature remains neutral with regard to jurisdictional claims in published maps and institutional affiliations.

1 Department of Process Engineering and Applied Science, Dalhousie University, Halifax, Canada
Mumuni Amadu

mm846771@dal.ca

Michal J. Pegg

Michael.Pegg@dal.ca

\section{Affiliations}

\section{Mumuni Amadu' ${ }^{1}$ Michal J. Pegg ${ }^{1}$}

